\title{
Diabetes Mellitus Gestacional: uma revisão narrativa
}

\author{
Gestational Diabetes Mellitus: a narrative review
}

Diabetes Mellitus Gestacional: una revisión narrativa

Ana Carolina Valadão Oliveira ${ }^{1 *}$, Otávio Benedito Rodrigues Guerra da Silva ${ }^{1}$, Lucas Barandas Souza ${ }^{1}$, Bárbara Bertolino Ravagnani ${ }^{1}$, Luana Carolina Rodrigues Guimarães ${ }^{1}$, Isabela Barandas Souza ${ }^{1}$, Paula Ananda Chacon Inês².

\section{RESUMO}

Objetivo: Dado a complexidade da abordagem e divergência entre literaturas sobre Diabetes Mellitus Gestacional (DMG), os autores realizaram uma revisão bibliográfica abordando aspectos recentes relacionados a prevalência, rastreamento, prevenção e tratamento da referida patologia. Revisão bibliográfica: $\mathrm{O}$ DMG caracteriza-se por intolerância à glicose, podendo ocorrer em qualquer período da gestação e acarretar sérios danos. Ressalta-se a importância da manutenção do controle glicêmico evitando complicações na gestação. É necessário que desde o início da gestação seja realizado exame para o rastreamento da DMG, principalmente na $24^{\mathrm{a}}$ a $28^{\mathrm{a}}$ semana de gestação e em casos de diagnóstico positivo a primeira opção é o tratamento não medicamentoso, consistido de dieta e atividade física, não havendo melhora, o tratamento medicamentoso faz-se necessário, com uso de hipoglicemiantes orais ou insulinoterapia. Considerações finais: Diante das divergências existente no manejo da DMG, cabe ao profissional de saúde estar atualizado para realizar o acompanhamento das gestantes nas unidades básicas de saúde. Além disso, faz-se necessário a adesão de um consenso entre as literaturas para que se estabeleça um valor padrão melhorando a abordagem ao paciente.

Palavras-chave: Diabete gestacional, Diagnóstico, Tratamento.

\begin{abstract}
Objective: Given the complexity of the approach and the divergence between literature on Gestational Diabetes Mellitus (GDM), the authors carried out a bibliographic review addressing recent aspects related to the prevalence, screening, prevention and treatment of this pathology. Bibliographic review: GDM is characterized by glucose intolerance, which can occur at any time during pregnancy and cause serious damage. The importance of maintaining glycemic control is emphasized, avoiding complications during pregnancy. It is necessary that, from the beginning of pregnancy, an examination is carried out to screen for GDM, especially in the 24th to 28th week of pregnancy and in cases of positive diagnosis the first option is non-drug treatment, consisting of diet and physical activity, with no improvement, drug treatment is necessary, with the use of oral hypoglycemic agents or insulin therapy. Final considerations: In view of the divergences in the management of DMG, it is up to the health professional to be updated to monitor the pregnant women in the basic health units. In addition, it is necessary to adhere to a consensus among the literature in order to establish a standard value improving the approach to the patient.
\end{abstract}

Keywords: Gestational diabetes, Diagnosis, Treatment.

\section{RESUMEN}

Objetivo: Dada la complejidad del abordaje y la divergencia entre la literatura sobre Diabetes Mellitus Gestacional (DMG), los autores realizaron una revisión bibliográfica abordando aspectos recientes relacionados con la prevalencia, cribado, prevención y tratamiento de esta patología. Revisión bibliográfica: La DMG se caracteriza por la intolerancia a la glucosa, que puede ocurrir en cualquier momento durante el embarazo y causar daños graves. Se enfatiza la importancia de mantener el control glucémico evitando complicaciones durante el embarazo. Es necesario que, desde el inicio del embarazo, se realice un examen de cribado de DMG, especialmente en las semanas 24 a 28 de gestación y en los casos de diagnóstico

1 Universidade de Franca (UNIFRAN), Franca - SP. *E-mail: cvaladao03@gmail.com

${ }^{2}$ Centro Universitário Municipal de Franca (Uni-FACEF), Franca - SP.

SUBMETIDO EM: 3/2021 
positivo la primera opción es el tratamiento no farmacológico, consistente en dieta y fisioterapia. actividad, sin mejoría, es necesario el tratamiento farmacológico, con el uso de hipoglucemiantes orales o terapia con insulina. Consideraciones finales: Ante las divergencias en el manejo de la DMG, corresponde al profesional de la salud estar actualizado para monitorear a las gestantes en las unidades básicas de salud. Además, es necesario adherirse a un consenso entre la literatura para establecer un valor estándar que mejore el abordaje del paciente.

Palabras clave: Diabetes gestacional, Diagnóstico, Tratamiento.

\section{INTRODUÇÃO}

O Diabetes Mellitus (DM) é uma doença que cresce gradativamente a cada ano, a estimativa da DM é de 14,3 milhões de adultos entre 20 e 79 anos (FEBRASGO, 2019). A DM pode ser definida como um distúrbio endócrino caracterizado pelos níveis elevados de glicose no sangue (maiores do que $100 \mathrm{mg} / \mathrm{dL}$ em jejum) denominado de hiperglicemia, podendo ser ocorrido pela deficiência insulínica. A insulina é um hormônio produzido e secretado pelo pâncreas, tem como função promover a entrada das moléculas de glicose nas células (ARAÚJO IM, et al., 2020).

O DM pode decorrer pela deficiência na produção e/ou liberação pancreática de insulina ou na resistência periférica da mesma, sendo classificadas, diante disto, em tipo 1 ou tipo 2, e pode manifestar-se em várias etapas da vida de um indivíduo. A DM tipo 1 distingue-se por acometer primariamente crianças e adolescentes, sendo um dos tipos mais agressivos. Ela ocorre como decorrência de um processo autoimune que destroem as células beta pancreáticas, fazendo com que o indivíduo não consiga produzir o hormônio insulina, tendo então a sua deficiência completa no organismo. Já a DM tipo 2 é reconhecida como uma doença multifatorial que afeta a qualidade de vida das pessoas acometidas, e é fisiologicamente caracterizada por uma resistência das células à insulina decorrente de uma falha na sua secreção pelo pâncreas (OLIVEIRA EC, et al., 2016; RODRIGUES BSSL, et al., 2019).

O Ministério da Saúde (MS), a Sociedade Brasileira de Diabetes (SBD) e a Federação Brasileira das Associações de Ginecologia e Obstetrícia (FEBRASGO) trazem que quando a hiperglicemia inicia ou tem seu primeiro reconhecimento durante a gestação, a DM passa a ser denominada de Diabetes Mellitus Gestacional (DMG) que é uma condição representada por intolerância à glicose em vários níveis de intensidade, que podem ou não persistir após o parto. Um outro diagnóstico possível e importante de ser feito e diferenciado deste citado anteriormente é o Diabetes Mellitus diagnosticado na gestação, sendo está detectada quando a mulher já tinha DM, porém sem diagnóstico, e durante a gestação apresenta hiperglicemia com níveis de glicemia atingindo critérios para DM na ausência de gravidez, segundo a Organização Mundial da Saúde (SILVA RR, et al., 2019; BARROS GM, 2017).

O Diabetes Mellitus Gestacional é um problema prevalente no mundo, e ocorre em gestantes que não possuem a capacidade de aumentar a produção de insulina o suficiente para neutralizar a resistência insulínica provocada pelos hormônios diabetogênicos produzidos durante a gestação, como lactogênio placentário, cortisol e prolactina e, pelas mudanças no controle glicêmico devido a própria gestação. A presença desta patologia durante a gestação pode ocasionar muitos efeitos indesejáveis, tanto para a mãe quanto para o feto (ALBRECHT CC, et al., 2019; ORGANIZAÇÃO PAN AMERICANA DA SAÚDE, 2016; RODRIGUES BSSL, et al., 2019).

Conforme a FEBRASGO (2019) traz em seu estudo, aproximadamente um em cada seis nascimentos são de mulheres com hiperglicemia durante a gestação, destas com $84 \%$ decorrentes de DMG, uma porcentagem para qual devemos ficar atentos, uma vez que, o aumento da glicemia materna traz sérias consequências que podem pôr o feto e a mãe em risco. Algumas complicações mais frequentemente associadas ao DMG são, por exemplo, a cesariana, a pré-eclâmpsia e o risco de DM pós-parto para a mãe, e a prematuridade, a macrossomia, a distocia de ombro, a hipoglicemia e a morte perinatal para o concepto. Além disso, estimase que a prevalência de atendimentos no Sistema Único de Saúde (SUS) de mulheres com Diabetes Mellitus Gestacional é de 18\% se utilizado os critérios diagnósticos atuais (OLIVEIRA LC, et al., 2019; GUERRA JVV, et al., 2019; GUERRA JVV, et al., 2019; SOCIEDADE BRASILEIRA DE DIABETES, 2020). 
Atualmente, tem-se o conhecimento que a DMG constitui um problema de saúde pública, pois apresenta elevadas taxas de morbidade perinatal, podendo chegar a aproximadamente $45 \%$. Vários estudos destacam a importância do controle glicêmico antes e durante a gestação, pois este controle diminui significativamente esta mortalidade/morbidade perinatal e consequentemente as malformações congênitas (QUEIROZ IS, et al., 2019).

Uma gestação que cursa com Diabetes Mellitus Gestacional (DMG) é classificada como alto risco e uma classificação da gestante de forma errônea contribui para elevação da morbimortalidade perinatal. Diante disto, preconiza-se o rastreamento e a busca ativa das gestantes com DMG, por meio de testes de glicemia e de sobrecarga de glicose, conforme indicação a partir da idade gestacional na qual a grávida se encontra. Em decorrência das graves complicações materno-fetais ocasionadas pela DMG, cada vez mais se preconiza o rastreio precoce, logo na primeira consulta de pré-natal, para que o tratamento seja prontamente instituído. (QUEIROZ IS, et al., 2019; WEINERT LS, et al., 2011).

Em seu estudo, Guerra JVV, et al. (2019) afirma que a reeducação alimentar na vida da gestante é de grande importância, sendo necessário o acompanhamento de um profissional capacitado, pois, sabe-se que tal conduta pode contribuir na prevenção e melhora da saúde tanto da mulher, como do concepto. A primeira opção terapêutica diante da DMG é o tratamento não medicamentoso, ou seja, reeducação alimentar como supracitado, que atrelada a prática de atividades físicas, na ausência de contraindicações, vem se tornando um dos elementos mais importantes. Contudo, o seguimento contínuo da gestante se faz necessário, pois diante da falha desta primeira modalidade terapêutica, se faz necessário a introdução do tratamento medicamentoso (OLIVEIRA AC, et al., 2016; MORAIS AM, et al., 2019).

Levando em consideração a complexidade associada à doença, há um consenso entre os autores em destacar que todas as gestantes devem ser rastreadas. Tendo em vista este fator, cabe ao médico acompanhar o controle glicêmico destas pacientes e ter como objetivo principal o rastreamento precoce dos casos de Diabetes Mellitus Gestacional para planejamento de ações, que nestes casos, possam diminuir os índices de complicações. Assim, como o conhecimento amplo do ponto de vista que envolvem a doença, o propósito deste estudo foi avaliar os aspectos epidemiológicos vinculados a prevalência, rastreamento/teste de diagnóstico e tratamento.

\section{REVISÃO BIBLIOGRÁFICA}

\section{Prevalência}

A DMG é um problema de saúde prevalente em todo o mundo, estimando que se atinja aproximadamente $25 \%$ das gestantes, segundo dados as Sociedade Brasileira de Diabetes (ARAÚJO IM, et al., 2020). Nos Estados Unidos, a estimativa é de que $2 \%$ a $10 \%$ das gestantes sejam acometidas, e aproximadamente $4 \%$ das gestações ocorrem com complicações, computando um total de 135.000 casos a cada ano (ALMEIDA CAPL, et al., 2019).

Ao analisar a variável prevalência da DMG, estudos demonstram que a porcentagem gira em torno de $7,6 \%$ entre as mulheres maiores de 20 anos também atendidas no SUS, e a prevalência de pacientes com estado hiperglicêmico varia de 1\% a 37\%, tendo uma média mundial de 16,2\%. Enfatiza-se que por ser uma patologia com alta prevalência, há uma necessidade de atualização constante e frequente destes dados (QUEIROZ IS, et al., 2019; ALMEIDA CAPL, et al., 2019; MORAIS AM, et al., 2019; GUERRA JVV, et al., 2018; FEBRASGO, 2019). .

\section{Fatores de risco}

Sabe-se que, por meio dos artigos publicados acerca do tema, o desenvolvimento de DMG está relacionado com a presença de diversos fatores, como geográficos, étnicos e raciais, e que existem fatores de risco ligados diretamente com a patologia, sendo eles o índice de massa corporal elevado, sobrepeso e obesidade, histórico familiar, idade materna avançada e hipertensão (GUERRA JVV, et al., 2018; FILHO LCS, et al., 2018). 


\section{Rastreamento e diagnóstico}

Estudos mais recentes têm demostrado que antecipar o rastreamento de DMG pode estar diretamente relacionado a desfechos mais favoráveis. Existem recomendações da utilização do Teste Oral de Tolerância a Glicose (TOTG) entre $18^{\underline{a}}$ a $20^{\underline{a}}$ semana de gestação, entretanto, não foi comprovado que o diagnóstico precoce possui relevância na incidência de complicação tanto maternas quanto fetais (FILHO LCS, et al., 2018).

A maioria dos autores, assim como o Ministério da Saúde e Organização Mundial da Saúde, recomendam que o período do rastreamento deve ser realizado na primeira consulta de pré-natal e a repetição entre a $24^{\mathrm{a}}$ a $28^{\mathrm{a}}$ semana de gestação, e apenas uma pequena parcela sugerem que o rastreamento deve ser iniciado na $20^{\mathrm{a}}$ semana de gestação. É interessante ressaltar que o Ministério da Saúde preconiza a realização do rastreamento para todas mulheres durante a gestação (BARROS GM, 2017; ORGANIZAÇÃO PAN AMERICANA DA SAÚDE, 2016; BOZATSKI BL, et al., 2019).

O diagnóstico de DMG é feito de diferentes maneiras a depender do país em que se encontra. Em geral, há duas fases: a triagem e a confirmação diagnóstica. Os métodos utilizados citados entre os autores são: a Glicemia de Jejum (GJ), pós-prandial e Teste Oral de Tolerância a Glicose (TOTG) e alguns ainda trazem o uso da hemoglobina glicada ( $\mathrm{HbA} 1 \mathrm{c})$; tomando como base os artigos selecionados, pode-se notar que há algumas divergências quanto aos valores exatos para o diagnóstico da DMG, divergência essa que dificulta o trabalho dos profissionais da saúde, pois se não houver um consenso, o diagnóstico fica falho, até podendo diminuir a taxa de prevalência da DMG, o que se tornaria um dado irreal (MORAIS AM, et al., 2019; MEIRA TB, 2018; FEBRASGO, 2019).

Quando observado os valores propostos para o diagnóstico de DMG por O'Sullivan JB e Mahan CM (1964), temos os seguintes parâmetros: Glicemia de Jejum de $90 \mathrm{mg} / \mathrm{dL}$, glicemia em 1 hora de $165 \mathrm{mg} / \mathrm{dL}$, glicemia em 2 horas de $143 \mathrm{mg} / \mathrm{dL}$ e glicemia em 3 horas de $127 \mathrm{mg} / \mathrm{dL}$. Com o objetivo de facilitar seu uso na prática, foi validado como critério que gestantes com dois ou mais valores alterados deveriam receber o diagnóstico de DMG e arredondado os valores para: GJ de $90 \mathrm{mg} / \mathrm{dL}$, $1 \mathrm{~h}$ de $165 \mathrm{mg} / \mathrm{dL}$, $2 \mathrm{~h}$ de $145 \mathrm{mg} / \mathrm{dL}$ e $3 \mathrm{~h}$ de 125 $\mathrm{mg} / \mathrm{dL}$ (FEBRASGO, 2019).

No Brasil, dentro das próprias recomendações do Ministério da Saúde há divergências, em que se encontra recomendações que durante o rastreio de DMG, um valor de Glicemia de Jejum maior que $85 \mathrm{mg} / \mathrm{dL}$ e TOTG maior que $110 \mathrm{mg} / \mathrm{dL}$ confirmam DMG; e valores de GJ maiores que $110 \mathrm{mg} / \mathrm{dL}$, após dois exames, confirma o DMG em qualquer fase da gestação (MORAIS AM, et al., 2019; ORGANIZAÇÃO PAN AMERICANA DA SAÚDE, 2016).

Em outros locais do próprio Ministério da Saúde, encontra-se recomendações que GJ maior ou igual a 95 $\mathrm{mg} / \mathrm{dl}$ e menor que $126 \mathrm{mg} / \mathrm{dl}$, junto com a presença de fatores de risco tem-se o diagnóstico da doença e, quando não presente os fatores de risco, faz-se necessário a realização do TOTG para confirmar o diagnóstico (GUERRA JVV, et al., 2019). Ciente da grande diferença de acessibilidade aos exames em todo território nacional, o próprio Ministério da Saúde apresenta informações que, na necessidade de realização da TOTG conforme suas recomendações, porém a indisponibilidade do mesmo, é aceitável repetir o exame de glicemia de jejum entre $24^{\mathrm{a}}$ e $28^{\mathrm{a}}$ semanas (HENNIGEN AW, et al., 2018).

A fim de uma melhor compreensão Hennigen AW, et al. (2018), traz em seu estudo que a International Association of Diabetes and Pregnancy Study Groups (IASDPG) recomenda que se durante o primeiro trimestre a GJ for menor que $92 \mathrm{mg} / \mathrm{dl}$, a gestante deve fazer o TOTG com $75 \mathrm{~g}$ de glicose para glicemia em jejum, 1 hora e 2 horas entre $24^{\underline{a}}$ e $28^{\text {a }}$ semanas de gestação. Para o diagnóstico da DMG é necessária uma Glicemia de Jejum igual ou superior a $92 \mathrm{mg} / \mathrm{dL}$ em qualquer momento da gestação ou alterações do TOTG, com glicemia de jejum maior que $92 \mathrm{mg} / \mathrm{dL}$, $1 \mathrm{~h}$ maior que $180 \mathrm{mg} / \mathrm{dL}$ e $2 \mathrm{~h}$ maior que $153 \mathrm{mg} / \mathrm{dL}$, valores esses que também estão sendo seguidos pela American Diabetes Association (ADA) (FILHO LCS, et al., 2018).

Ao observarmos os critérios propostos pela Organização Mundial da Saúde (OMS), já notamos uma divergência, visto que esse órgão considera o diagnóstico de DMG com pelo menos um dos valores alterado em qualquer fase da gestação. Os parâmetros utilizados são: GJ entre 92 e $125 \mathrm{mg} / \mathrm{dl}$, TOTG com glicose de $1 \mathrm{~h}$ maior ou igual a $180 \mathrm{mg} / \mathrm{dl}$ ou 2 horas entre 153 e $199 \mathrm{mg} / \mathrm{dl}$ (FEBRASGO, 2019; ZUCCOLOTTO DCC, et al., 2019; HENNIGEN AW, et al., 2018). 
Em contrapartida, a Organização Pan Americana da Saúde, em seu documento de Rastreamento e Diagnóstico do Diabetes Mellitus Gestacional, traz que para o diagnóstico sejam realizadas quatro coletas do TOTG com sobrecarga de $100 \mathrm{~g}$ de glicose e como pontos de corte valores de: Glicemia de Jejum (tempo zero) de $90 \mathrm{mg} / \mathrm{dL}$, $1 \mathrm{~h}$ (tempo 60 minutos) de $165 \mathrm{mg} / \mathrm{dL}$, 2h (tempo 120 minutos) de $145 \mathrm{mg} / \mathrm{dL}$ e 3h (tempo 180 minutos) de $125 \mathrm{mg} / \mathrm{dL}$, sendo necessário dois ou mais valores maiores ou iguais os citados acima (ORGANIZAÇÃO PAN AMERICANA DA SAÚDE, 2017).

Do mesmo modo, a FEBRASGO (2019) em seu manual de Rastreamento e diagnóstico de DMG no Brasil, sugere-se que, estratégias sejam levadas em consideração para que possamos ter o diagnóstico da DMG. Com isso, devemos considerar as particularidades do nosso pais, levando em consideração a situação de viabilidade financeira e a disponibilidade técnica das regiões, podendo sê-la total ou parcial, com a finalidade de que, todas as gestantes possam realizar o exame proposto.

Nessa premissa, caso haja viabilidade financeira na região é indicado que se realize a glicemia de jejum, se valor menor que $92 \mathrm{mg} / \mathrm{dL}$, é indicado que o próximo teste seja o TOTG com $75 \mathrm{~g}$ de glicose, ao menos um valor de: Jejum: 92 a $125 \mathrm{mg} / \mathrm{dL} ; 1^{\text {a }}$ hora $\geq 180 \mathrm{mg} / \mathrm{dL}$; $2^{\text {a }}$ hora: 153 a $199 \mathrm{mg} / \mathrm{dL}$ assegura o diagnóstico de DMG. Caso não haja viabilidade financeira na região é indicado que, se glicemia de jejum alterada, indica-se a realização de um novo teste de glicemia de jejum entre a $24^{\underline{a}}$ a $28^{\underline{a}}$ semana de gestação, e se valor for entre 92 a $125 \mathrm{mg} / \mathrm{dL}$ confirma o diagnóstico de DMG (FEBRASGO, 2019).

\section{Tratamento}

Assim como nos outros tipos de diabetes, o tratamento do DMG tem como primeira opção uma dieta individualizada e prática de atividade física, quando ausente as contraindicações. Para uma adequada prática de atividade física, é recomendado atividades aeróbicas sem risco e de baixo impacto, como caminhadas e natação, com intensidade baixa a moderada, por 30 a 45 minutos, três vezes na semana. A elaboração da dieta tem como finalidade um adequado controle metabólico, visando uma devida nutrição materno-fetal (BOZATSKI BL, et al., 2019; TUON RA, et al., 2016; OLIVEIRA AC, et al., 2016).

Aproximadamente 15\% das gestantes com DMG não conseguem alcançar o controle glicêmico após duas semanas, e, quando não alcançado as metas propostas, o tratamento farmacológico é iniciado, o qual tem como recomendação de primeira linha pela Sociedade Brasileira de Diabetes, a insulinoterapia subcutânea, sendo as mais utilizadas a Neutral Protamine Hagedorn (NPH) e a regular. Entretanto, o uso desta é complexo e de menor aceitação pelas pacientes e, embora alguns autores orientam a descontinuação do uso de antidiabéticos orais, outros autores trazem o uso destes como uma alternativa, tendo seus maiores exemplos a glibenclamida e metformina, sendo que ambas se mostraram, recentemente, de efetividade comparável à da insulina, porém tendo o uso da metformina mais prevalente na prática por ser mais segura (GUERRA JVV, et al., 2018; MEIRA TB, 2018; MORAIS AM, et al., 2019; BEZERRA SJ e CARLOTTO SM, 2019; RODRIGUES BSSL, et al., 2019).

A partir do momento que se institui o tratamento da DMG, tem que se ter em mente o controle glicêmico e sua reavaliação constante pelo médico a cada 2 semanas até 36 semanas de gestação e semanalmente após este período, além de orientar a gestante e motiva-la a realizar glicemias capilares. A periodicidade das monitorizações da glicemia capilar são controvérsias conforme a fonte bibliográfica, sendo que algumas orientam medidas em jejum e após as três principais refeições, enquanto outras recomendam 7 medidas diárias, incluindo jejum, antes e após todas as principais refeições. Ademais, estudos mostram que a partir da evidência de bom controle após 1 a 2 semanas, pode-se espaçar a monitorização para dias alternados, sendo preconizado 4 vezes na semana (HENNIGEN AW, et al., 2018; MEIRA TB, 2018).

Assim como no rastreamento e o diagnóstico, o controle glicêmico apresenta algumas pequenas variações de valores considerados adequados, a depender da fonte estudada, tendo-se como referências: Glicemia de Jejum entre $60 \mathrm{mg} / \mathrm{dL}$ e $90 \mathrm{mg} / \mathrm{dL}$ ou entre $65 \mathrm{mg} / \mathrm{dL}$ e $95 \mathrm{mg} / \mathrm{dL}$ ou até mesmo entre $80 \mathrm{mg} / \mathrm{dL}$ e $110 \mathrm{mg} / \mathrm{dL}$; e de glicemia pós-prandial (1h) entre $100 \mathrm{mg} / \mathrm{dL}$ e $120 \mathrm{mg} / \mathrm{dL}$ ou até $140 \mathrm{mg} / \mathrm{dL}$; e $2 \mathrm{~h}$ de até $120 \mathrm{mg} / \mathrm{dL}$ ou até $155 \mathrm{mg} / \mathrm{dL}$. É importante lembrar que em mulheres com risco aumentado de hipoglicemia, esses alvos devem ser aumentados para GJ até $99 \mathrm{mg} / \mathrm{dl}$ e ao deitar-se de $80 \mathrm{mg} / \mathrm{dL}$ até $120 \mathrm{mg} / \mathrm{dl}$. Quando avaliado a 
hemoglobina glicada $(\mathrm{HbA} 1 \mathrm{c})$ na primeira consulta, é orientado que a mesma seja repetida mensalmente até que a gestante alcance valores menores que 6\%, quando então pode se realizar a avaliação a cada 2 ou 3 meses, a critério do médico que a acompanha (ORGANIZAÇÃO PAN AMERICANA DE SAÚDE, 2019; FERREIRA AF, et al., 2018; MEIRA TB, 2018).

Diante disto, para que se atinja a promoção de saúde e prevenção de doenças materno-fetais, é importante que se tenha educação em saúde focado nas questões do dia-a-dia, baseado na realidade e na valorização da autonomia, associado a confirmação pelos profissionais de saúde do entendimento, por parte das gestantes, de todas as informações disponibilizadas para as mesmas e se elas alteraram seus hábitos após reconhecimento da patologia (OLIVEIRA LC, et al., 2019).

\section{CONSIDERAÇÕES FINAIS}

Portanto, considerando que o Diabetes Mellitus Gestacional representa uma das situações de alto risco durante a gestação, é evidente a necessidade da intervenção do profissional e/ou agente de saúde para favorecer, o rastreamento, diagnóstico e cuidados com as pacientes para que haja um bom acompanhamento do pré-natal, garantindo uma assistência de qualidade ao longo da gestação. Contudo o presente estudo demonstrou que há divergência quanto aos valores padrões utilizados para realizar o rastreamento e o diagnóstico do DMG, o que dificulta quanto ao controle da mesma. No entanto, é visto que, faz-se necessário a adesão de um consenso entre as literaturas para que se estabeleça um valor padrão, com intuito de auxiliar no controle adequado da glicemia durante a gestação e reduzir os riscos perinatais relacionados à doença.

\section{REFERÊNCIAS}

1. ALBRECHT CC, et al. Características evidenciadas em recém-nascidos de gestantes hipertensas e diabéticas: revisão sistemática da literatura. J. nurs. Health., 2019; 9(1): 1-20.

2. ALMEIDA CAPL, et al. O enfermeiro docente e o diabetes mellitus gestacional: o olhar sobre a formação. Enfermagem Foco, 2019; 10(1): 111-116.

3. ARAÚJO IM, et al. Cuidados de enfermagem à pacientes com diabetes mellitus gestacional. Revista Brasileira Interdisciplinar de Saúde, 2020; 2(1): 43-8.

4. BARROS GM. Fatores de risco para o Diagnóstico de Enfermagem Risco de Glicemia Instável em gestantes Instrumento de classificação: estudo caso controle. 2017. 94 f. Dissertação (Mestrado Profissional em Enfermagem Assistencial) - Escola de Enfermagem Aurora de Afonso Costa, Niterói, 2017; 94 p.

5. BEZERRA SJ, CARLOTTO SM. Diabetes Mellitus Gestacional. Rev. Saberes da Faculdade São Paulo. $2019 ; 9$ : 1.

6. BOZATSKI BL, et al. Perfil epidemiológico de gestantes diabéticas no município de Itajaí, SC. Arquivo Catarinense Med., 2019; 48(2): 34-55.

7. FEDERAÇÃO BRASILEIRA DAS ASSOCIAÇÕES DE GINECOLOGIA E OBSTETRÍCIA (FEBRASGO). Rastreamento e diagnóstico de diabetes mellitus gestacional no Brasil. Femina, 2019; 47(6): 786-796.

8. FERREIRA AF, et al. Diabetes gestacional: serão os atuais critérios de diagnóstico mais vantajosos?. Acta Med Port, 2018; 31(7-8): 416-424.

9. FILHO LCS, et al. Impacto no trimestre de diagnóstico no Diabetes Mellitus Gestacional no tratamento utilizado e na classificação de peso do recém-nascido. ACM arquivo catarinense med., 2018; 47(2): 137-146.

10. GUERRA JVV, et al. Diabetes gestacional e assistência pré-natal no alto risco. Revista enferm. UFPE on line, 2019; 13(2): 449-454.

11. GUERRA JVV, et al. Diabetes gestacional e estado nutricional materno em um hospital universitário de Niterói. J. nurs. Health, 2018; 8(1): 1-11.

12. HENNIGEN AW, et al. Tele Condutas: diabetes e gestação. Porto Alegre; Universidade Federal do Rio Grande do Sul. Telessaúde, 2018.

13. MEIRA TB. Instrumento para o cuidado de mulheres gestantes com diabetes mellitus baseado no modelo Sunrise. Rio de Janeiro, 2018; s.n; 75.

14. MOURA BLA, et al. Internações por complicações obstétricas na gestação e desfechos maternos e perinatais, em uma coorte de gestantes no Sistema Único de Saúde no Município de São Paulo, Brasil. Cad. Saúde Pública, 2018; 34(1).

15. MORAIS AM, et al. Perfil e conhecimento de gestantes sobre o diabetes mellitus gestacional. Revista epidemiol. controle infecção, 2019; 9(2): 134-141.

16. O'SULLIVAN JB, MAHAN CM. Criteria for the oral glucose tolerance test in pregnancy. Diabetes, 1964; 13:278-85.

17. OLIVEIRA AC, et al. Estudo da relação entre ganho de peso excessivo e desenvolvimento de diabetes mellitus e doença hipertensiva específica na gestação. J. Health Sci. Inst., 2016, 34(4): 231-239.

18. OLIVEIRA EC, et al. Diabete mellitus gestacional: uma revisão de literatura. Ver. Cien. FacMais, 2016; 5(1): $128-140$.

19. OLIVEIRA LC, et al. Auditoria de um serviço de atendimento de gestantes portadoras de Diabetes mellitus gestacional. Saúde e Pesquisa, 2019; 12(3): 513-520. 
20. ORGANIZAÇÃO PAN-AMERICANA DA SAÚDE (OPAS). Rastreamento e diagnóstico de diabetes mellitus gestacional no Brasil. Brasília, DF: OPAS, 2016.

21. ORGANIZAÇÃO PAN-AMERICANA DA SAÚDE (OPAS). Tratamento do diabetes mellitus gestacional no Brasil. Brasília, DF: OPAS, 2019.

22. QUEIROZ IS, et al. Complicações e doenças pré-existentes em gestantes com diabetes mellitus. Revista enferm. UFPE on line, 2019; 13(5): 1202-1207.

23. RODRIGUES BSSL, et al. Diabetes mellitus gestacional: Uma revisão sistemática sobre o tema. Braz. J. Surg. Clin. Res., 2019, 28(2): 20-24.

24. SILVA RR, et al. Desfechos materno-fetais de gestantes com e sem diabetes mellitus gestacional. ACM arq. catarinense med., 2019; 48(3):79-92.

25. SOCIEDADE BRASILEIRA DE DIABETES. Diretrizes da Sociedade Brasileira de Diabetes 2019-2020. São Paulo: Editora Clannad., 2020; p. 279-288.

26. TUON RA, et al. Impacto do monitoramento telefônico de gestantes na prevalência da prematuridade e análise dos fatores de risco associados em Piracicaba, São Paulo, Brasil. Cad. saúde pública., 2016; 32(7).

27. WEINERT LS, et al. Diabetes gestacional: um algoritmo de tratamento multidisciplinar. Arq Bras Endocrinol Metab., $2011 ; 55(7): 435-445$.

28. ZUCCOLOTTO DCC, et al. Padrões alimentares de gestantes, excesso de peso materno e diabetes gestacional. Revista Saúde Pública, 2019; 53(52): 1-12. 